\title{
LA AUTOMATIZACIÓN EN EL SECTOR TERCIARIO: evolución del service encounter
}

\section{1- Eva Diz-Comesaña*}

Doctora en Administración y Dirección de Empresas por la Universidad de Vigo (UVIGO), Espanha.

Profesora del Departamento de Oganización de Empresas y Marketing de la Universidad de Vigo - UVIGO, Espanha. evadiz@uvigo.es

\section{2- Begoña Urgal González}

Doctora en Administración y Dirección de Empresas por la Universidad de Vigo (UVIGO), Espanha.

Profesora del Departamento de Oganización de Empresas y Marketing de la Universidad de Vigo - UVIGO, Espanha. burgal@uvigo.es 


\section{LA AUTOMATIZACIÓN EN EL SECTOR TERCIARIO: EVOLUCIÓN DEL SERVICE ENCOUNTER}

\section{RESUMEN}

Tradicionalmente, la mayoría de los servicios han sido el resultado de procesos que se realizan en su mayor parte, sino en su totalidad, en contacto directo entre el cliente y los representantes de la empresa prestataria del servicio, en un contexto organizativo determinado. No obstante, en la actualidad muchos investigadores han reconocido la importancia de la tecnología en la prestación del servicio. Desde esta perspectiva, en este trabajo se analizan los distintos elementos que intervienen en el service encounter y su evolución ante la incorporación de procesos automatizados. Para ello, se ha realizado un análisis empírico sobre cuatro subsectores- autopistas, aparcamientos, lavado de automóviles y alquiler de películas cinematográficas- donde se pone de manifiesto cierta evolución en los elementos que participan en la prestación del servicio y en las relaciones que éstos mantienen entre sí, y se deja intuir que la implantación de tales procesos podría permitir alcanzar cotas aceptables de productividad y flexibilidad, variables tradicionalmente antagónicas.

\section{Palabras clave}

Servicios, Service Encounter, Automatización.

\section{AUTOMATION IN TERTIARY SECTOR: EVOLUTION OF SERVICE ENCOUNTER}

\section{ABSTRACT}

Traditionally, the majority of the services have been the result of process mainly made in direct contact between customers and employees of the firm that is making the service. However, many researchers have recognized nowadays the importance of technology in the service delivery. Since this perspective, this work analyses the different elements of the service encounter and its evolution in front of incorporation of automated process. For this, we have realized an empirical study on four industries, namely, toll motorways, car parks, carwash and video/DVD rental companies. This study gave us the opportunity to see certain evolution in the elements that take part in the service encounter and in the relationships that these have between themselves. Would seem to indicate that the incorporation of these processes could allow acceptable levels of productivity and flexibility, traditionally considered antagonistic variables.

\section{Keywords}

Services, Service Encounter, Automation. 


\section{Introduccción}

Todo servicio posee un valor, pero éste solamente se hará efectivo cuando los recursos materiales y humanos pertenecientes a la empresa de servicios estén disponibles, y cuando el cliente sienta la necesidad y acudiendo a la empresa, la satisfaga (Eiglier y Langeard, 1993, p. 3). En definitiva, el valor del servicio se manifiesta cuando tiene lugar la producción del mismo. En este sentido, se ha considerado que la mayoría de los servicios son el resultado del contacto entre el cliente y los representantes de la empresa (personal en contacto), bajo el marco de la empresa prestataria del servicio (Bateson, 1985a; Solomon, Surprenant, Czpiel, \& Gutman, 1985; Norman, 1990; Bitner, Booms, \& Tetreault, 1990), dando lugar a lo que se ha llamado Service Encounter.

Diversos investigadores han analizado la interacción que se produce entre el cliente y los empleados en el service encounter (Bowen \& Schneider, 1985; Solomon et al., 1985; Surprenant \& Solomon, 1987; Bitner et al., 1990; Lewis \& Entwistle, 1990; Rafaeli, 1993; Mohr \& Bitner, 1995; Price, Arnould, \& Deibler, 1995; Bettencourt \& Gwinner, 1996; Clemmer \& Schneider, 1996; Goodwin, 1996; Goodwin \& Gremler, 1996; Hartline \& Ferrell, 1996; Fischer, Gainer, \& Bristor, 1997; Gremler, Gwinner, \& Brown, 2001; Patterson \& Baron, 2010; Wägar \& Lindqvist, 2010).

No obstante, en las últimas décadas se ha producido en un gran número de relaciones de servicio una sustitución total o parcial del personal en contacto por medios tecnológicos. La continua proliferación de tecnologías self-service pone de manifiesto la importancia que ha adquirido la tecnología en el service encounter. Aunque diversos investigadores han contribuido al estudio de la repercusión que tiene la tecnología en la prestación del servicio (Globerson \& Maggard, 1991; Fisk, Brown, \& Bitner, 1993; Schneider \& Bowen, 1995; Dabholkar, 1996a; Dabholkar, 1996b; Lawrence \& Karr, 1996; Parasuraman, 1996; Quinn, 1996; Harvey, Lefebvre, \& Lefebvre, 1997; Meuter \& Bitner, 1998; Bitner, Brown, \& Meuter 2000; Bowden, 2002; Ba, Stallaert, \& Zhang, 2010) sigue existiendo un vacío en este campo. La preocupación fundamental ha sido el estudio de la reacción del cliente, siendo escasos los trabajos que analizan el efecto que la incorporación de la tecnología puede tener en todos los elementos que intervienen en el service encuounter, esto es, no sólo en el cliente, sino también en el personal en contacto y en la organización.

Por ello, el objetivo de este trabajo es estudiar cómo evolucionan todos los elementos que intervienen en el service encounter (clientes, personal en contacto y organización) y las relaciones que éstos mantienen entre sí con la incorporación de sistemas automatizados. Para ello se centra la atención en interacciones aisladas que podrían formar parte de una cascada de encuentros².

Para cumplir con este cometido se ha realizado un análisis empírico sobre empresas ubicadas en territorio español, pertenecientes al subsector autopistas, aparcamientos, lavado de automóviles o alquiler de películas cinematográficas, y que en el año 2000 han alcanzado un nivel de facturación superior o igual a 300.000 euros.

Este trabajo se estructura en cuatro secciones. A continuación de la introducción (sección 1), en la sección 2, se recogen los fundamentos teóricos relativos a la posible evolución de los elementos del service encounter ante la automatización. En la sección 3 se incluye el análisis empírico y, finalmente, en la sección 4, las principales conclusiones del estudio.

\section{Elementos que Participan en el Service Encounter}

Tal como se ha indicado, para la prestación de un servicio se necesitan tres elementos: cliente, personal en contacto y organización, cuyas interacciones constituyen lo que se ha dado en llamar service encounter.

Por otro lado, en muchas ocasiones la prestación del servicio tiene lugar ante la presencia de otros clientes, pudiendo éstos tener una importancia clave. Por tanto, se ha considerado apropiado, siguiendo a Martin y Pranter (1989) y Grove y Fisk (1997), analizar también las interacciones que tienen lugar entre los consumidores en el establecimiento del servicio.

Todo ello ha llevado a considerar, siguiendo a Eiglier y Langeard (1993), como elementos del subsistema de operaciones de las empresas de servicios, los siguientes: el personal en contacto, el soporte físico, el sistema de organización interna -estos tres pertenecientes a la empresa-, el cliente, los otros clientes -éstos pertenecientes al mercado- y los servicios -resultantes de la interacción entre los clientes y los elementos de la empresa- (Diz, 2005, p. 682). 
Una vez concretados los elementos que intervienen en la prestación del servicio se ha creído conveniente realizar ciertas puntualizaciones sobre cada uno de ellos.

\subsection{Cliente}

El cliente resulta de vital importancia en el sistema de producción de las empresas de servicios, puesto que normalmente además de ejercer funciones de consumidor también actúa como coproductor.

Hasta hace poco las investigaciones en torno a este elemento se basaban sobre todo en el comportamiento de compra. Esto es así, hasta que se acepta que el cliente de una empresa de servicios debe ejercer, en la mayor parte de los casos, una función de productor. En este sentido, el auto-servicio se manifiesta como un claro ejemplo de este hecho, pero aunque no sea ésta la situación, el cliente normalmente deberá participar en la producción del servicio y en muchas ocasiones también en otras funciones, como por ejemplo, en el diseño y control del mismo.

Autores como Sasser et al. (1982) y Voss, Armistead, Johnston y Morris (1985) reconocen que el consumidor a menudo participa en el proceso productivo e interactúa con el proveedor del servicio. Berry (1981) indica que esa relación frente a frente es una importante oportunidad para la venta de otros bienes y servicios así como para el desarrollo de una imagen corporativa.

Por otro lado, Chase (1978, p.140) señala que el contacto con los consumidores reduce el nivel de eficiencia de la operación, por tanto, habrá que evitarlo en la medida de lo posible. Este autor indica que “cuanto menos se mezcle el cliente" en los asuntos de la empresa, de más libertad dispondrá ésta. Según Chase separar la producción del consumo optimiza la actividad. No obstante, con posterioridad defiende el enfoque basado en el contacto con el consumidor (Chase, 1992), ya que suele permitir la venta de productos adicionales (Chase \& Aquilano, 1994). Mills (1986), siguiendo esta perspectiva, indica que los problemas surgen porque resulta difícil predecir el comportamiento del consumidor con cierto grado de certeza. Sus trabajos se centran en el desarrollo de la idea de contrato entre el cliente y el personal, que provee un conjunto de reglas implícitas relativas al comportamiento del consumidor en el proceso. Por su parte, Czepiel, Solomon y Surprenant (1985) mantienen que "los roles del cliente y el proveedor están bien definidos".

Otros autores han desarrollado, además, su propio entendimiento del rol del consumidor en la operación de servicios. Por ejemplo, Langeard, Bateson, Lovelock y Eiglier (1981), Bateson (1985), y Eiglier y Langeard (1993) reconocen que el consumidor tiene un rol de producción al igual que de consumo.

Johnston (1989) indica que el consumidor, al igual que los empleados, es un participante activo en la creación, provisión y control de los servicios, desempeñado tareas relacionadas con la provisión de servicios para sí mismo, para otros consumidores -creando el ambiente de servicio y participando así en la formación de los mismos- y para la propia organización -proporcionando información para el control-.

Otro de los autores que ha descrito con mayor intensidad el concepto de participación del cliente, ha sido Toffler (1980), quien crea una nueva palabra, "prodsumidor" (prosumer), uniendo los vocablos productor y consumidor, obteniendo así un término que designa la integración progresiva que se manifiesta entre la función de producción y consumo.

En este sentido, Flipo (1993, p. 90) considera que:

El contacto comercial no reside únicamente en la sonrisa, o en la educación. Es sobre todo capacidad para la comunicación, es decir, sacar pleno partido de la participación del cliente en el proceso de producción del servicio para satisfacerlo. El problema organizativo que se plantea la empresa de servicios radica en facilitar esta comunicación, y además en la justa medida, es decir, evitando que el cliente se sienta satisfecho de forma genérica, más allá de lo que esperaba.

Por otro lado, Zeleny (citado en Norman, 1990) considera el auto-servicio como el único camino que permite mantener la eficacia cuando se trata de servicios intensivos en mano de obra y con un potencial limitado respecto a la mejora de la productividad. Este autor indica que en las sociedades occidentales siguiendo los cánones de la teoría económica clásica, el mecanismo de los precios para la mano de obra no funciona; ante esta situación, los costes se incrementarán con mayor rapidez que la productividad, no pudiendo el mercado soportar esta subida, prefiriendo así el auto-servicio. Lovelock y Young (1977, 1979) también abordan el tema desde la perspectiva de la empresa individual, considerando la participación del cliente como un medio que permite reducir los costes e incrementar la productividad. En este sentido, 
Bitner, Faranda, Hubebert y Zeithaml (1997) considera que el cliente crea calidad y productividad en las experiencias de servicio. Ante esta perspectiva, considerando al cliente como coproductor, hay autores como Martin, Horne y Chan (2001) que analizan la productividad del mismo como componente de la empresa.

No obstante, esta participación no siempre tiene lugar del mismo modo, ya que en algunos casos surge de forma espontánea por parte del cliente, mientras que en otros se necesita que la empresa proveedora lo motive para que éste participe, ya que esta colaboración suele resultar esencial. Por tanto, la empresa tratará de obtener cierto grado de cooperación por parte del cliente, presentando éste así dos dimensiones: una externa a la empresa y otra interna donde participa en las operaciones de la misma (Tat Keh \& Wei Teo, 2001; Hsieh, Yen, \& Chin, 2004).

Ante la bifuncionalidad que caracteriza al cliente, la empresa debe procurar el acoplamiento mutuo entre ambos. Para ello es importante adaptarse a los gustos y necesidades del consumidor, pero también tratar de dirigir la participación del mismo (Johnston, 1989; Czepiel, 1990).

Este enlace determina en parte el posicionamiento estratégico de la empresa, pudiendo establecer distintos modelos basados en las dimensiones que adoptan el personal y/o el equipo que se sitúa entre el cliente y la empresa (véase tabla 1 ).

Tabla 1

Tipos diferentes de acoplamiento mutuo entre la empresa y el cliente

\begin{tabular}{|c|c|c|}
\hline CLIENTE & Personal & Equipo \\
\hline Personal & Ej.: Formación convencional & $\begin{array}{c}\text { Ej.: Servicios de reparación } \\
\text { y mantenimiento }\end{array}$ \\
\hline Equipo & $\begin{array}{c}\text { Ej.: Cajero automático } \\
\text { instalado en un banco }\end{array}$ & $\begin{array}{c}\text { Ej.: Instalación automática } \\
\text { de lavado de automóviles }\end{array}$ \\
\hline
\end{tabular}

Nota. Fuente: Elaboración propia

Por otro lado, además de señalar los distintos tipos de acoplamiento entre la empresa y el cliente que propone Normann (1990), hay que decir que en ocasiones éstos no se manifiestan en estado puro sino que presentan distintas combinaciones.

En cualquier caso, la dimensión adquirida por el personal o el equipo en la relación empresa-cliente es una de las magnitudes a considerar que caracterizan dicha relación; pero existen muchas otras que también deben ser consideradas, tales como: la determinación de quien domina el proceso de acoplamiento y como lo hace, el grado de integración, el nivel de libertad de elección, aspectos relativos al tiempo y al espacio, etc.

\subsection{Personal en contacto y sistema de organización interna}

El personal de la empresa es el que determina el nivel de servicio alcanzado en el proceso de producción, de hecho son numerosos los autores, desde distintas posiciones o enfoques, que indican que el elemento básico de la calidad -de toda clase de calidad- es precisamente el factor humano. Bonoma (1987) señala que a medida que se incrementa la competencia global y los directivos tratan de buscar nuevos modelos y sistemas, es importante recordar que son las personas las que realizan el trabajo, y quienes no tienen esto en cuenta caerán en la mediocridad global. Otros autores como Huete, Roth y Vollmann (1988), una vez realizado un estudio empírico en el sector bancario americano, concluyen que por muy elevado que sea el nivel de industrialización de los servicios, el personal sigue resultando básico en la relación proveedor-cliente. Por otro lado, Bianco (1987) propone que aunque se admita la necesidad de la industrialización de los servicios, no puede ser cuestionado el papel que el ser humano desempeña junto a las máquinas.

Otros autores como Huselid (1995), Kalleberg y Moody (1994) destacan la importancia de la dirección de los recursos humanos. Maister (citado en Larrea Angulo, 1991) considera a los recursos humanos como el principal activo de una empresa y Marriot (citado en Larrea Angulo, 1991) indica que sin empleados satisfechos no es posible disponer de clientes satisfechos. También Schneider y Bowen (1985) ofrecen especial importancia a la idea de que cuando los empleados tienen opiniones favorables acerca del cuidado prestado a los recursos humanos por parte de la organización, los consumidores suelen percibir calidad en el servicio proporcionado. Esto implica que un programa de recursos humanos de una empresa puede ser 
un importante vehículo para dirigir tanto las necesidades del empleado como la calidad del output de la misma (Tansik, 1990). Esta relación entre empleado y consumidor considera el hecho de que ambos están física y psicológicamente muy cercanos durante la producción y entrega del servicio, de hecho, los empleados de empresas de servicios a menudo se identifican con el consumidor con el cual están interactuando.

Por otro lado, Carlzon (1988) considera la importancia de este factor cuando advierte que en su empresa tenían lugar aproximadamente 50 millones de encuentros de servicio (service encounter) o momentos de la verdad anualmente, en los cuales el cliente entra en contacto con los empleados. Además como Maister (1982a; 1982b; 1985) y Heskett $(1987$; 1988) han enfatizado, la relación empleado-cliente se convierte en un ente superior a la relación empresa-cliente, ya que en el encuentro del servicio, el personal en contacto es precisamente la empresa desde el punto de vista del cliente.

Por tanto, sobre el personal recae una enorme responsabilidad, lo que contrasta con la fragilidad del mismo. El factor humano suele llevar consigo cierto grado de riesgo, incertidumbre y problemática ante el aseguramiento de cierto nivel de calidad; frente a las máquinas que proporcionan precisión, regularidad y automatismo. Sin embargo, para conseguir los objetivos de satisfacción al cliente y rentabilidad, el empleado en ocasiones puede resultar imprescindible.

En definitiva, es importante que los empleados sean participativos, cooperativos, identificados, amantes de su trabajo, etc., cuestiones que suelen requerir una motivación de los mismos. Según autores como McGregor, las proposiciones realizadas por Herzberg (1977) todavía disponen de gran parte de vigencia. Este último indica que las variables de satisfacción o insatisfacción en el trabajo pueden resultar motivadoras o ergonómicas. Dentro de las primeras, entre las más importantes destaca la realización, el reconocimiento, el trabajo en sí y la responsabilidad, considerando estas variables como las generadoras de satisfacción. Respecto a los factores ergonómicos, resalta el sueldo, el status, las políticas de empresa y las condiciones de trabajo, calificando estos factores como los portadores de insatisfacción.

Por otro lado, González (1990a; 1990b) expone la siguiente ecuación: Motivación = Oportunidades + Capacidad. A la que Bernillón y Cerutti (1989) añaden un tercer elemento “querer, saber y poder participar". Querer participar requiere sensibilización hacia la calidad, comunicación en el interior de la empresa e implicación de los empleados en la vida de la misma. Saber participar supone formación. Y poder participar precisa estimular entre otros la creatividad, la iniciativa y la autonomía, actuando para ello sobre la estructura y dinámica de la empresa.

Juran (1990) considera que la motivación puede eliminar ciertos obstáculos para conseguir el liderazgo en calidad, tales como los siguientes:

- La ignorancia de los empleados que no se percatan de que están generando niveles de calidad inferiores.

- La prioridad de otros objetivos respecto a la calidad.

- La determinación de conflictos parciales que impiden optimizar el conjunto.

- La presencia de ciertos mitos culturales.

Tal como sugieren Eiglier y Langeard (1993) la motivación convierte a los empleados en intraempresarios. Además, indica Larrea (1991), hace posible la convergencia de intereses de la empresa, empleados y clientes.

Por otra parte, Chase (1985) indica que pueden distinguirse dos grupos de empleados: empleados de personas (front-office) y empleados de papel (back-office). Los primeros son los que conocemos como personal en contacto, y todo lo indicado anteriormente sobre la motivación debe considerarse todavía con mayor énfasis para este grupo.

El personal en contacto, como elemento del subsistema de operaciones de carácter interno, mantiene relaciones con el sistema de organización interna, donde le corresponde una posición jerárquica.

El análisis de los poderes internos no consiste únicamente en diferenciar los que dirigen de los que obedecen, de hecho, cada empleado tiene cierto grado de poder, y según como se ejerza, la empresa tendrá más o menos éxito.

Normalmente, el esquema de poder puede representarse por medio de un organigrama, situándose en la cima la dirección y en la base los trabajadores. Es la dirección la que debe arbitrar los conflictos relativos 
a la organización humana, sobre todo, mostrando las vías de interés general de la empresa contra los distintos intereses individuales, ya sean estos últimos relativos a un cliente, un departamento, etc.

En la actualidad, ante el elevado nivel de competitividad, la clientela es, o debiera ser, el punto de partida de cualquier estructura eficaz (Flipo, 1993). Aunque la empresa no tiene como único objetivo la satisfacción del consumidor, cada vez más, los demás objetivos (beneficio, crecimiento, etc.) dependen fundamentalmente del cumplimiento de éste.

Esto pone de manifiesto que el consumidor va a ejercer el poder dominante en la empresa, después de los propietarios o accionistas. Ya en 1978, Sasser et al. (citado en Flipo, 1993, pp. 128-129) proponen el siguiente plan de organización para una empresa de servicios (véase figura 1):

La parte superior de la tabla representa las necesidades de los clientes; y de esto dependen los demás elementos, sean materiales o humanos. En la parte central de la tabla se hallan los elementos técnicos necesarios para alcanzar el nivel de servicio fijado en un principio. Y en la parte inferior de la tabla se hallan los elementos que hacen funcionar el conjunto.

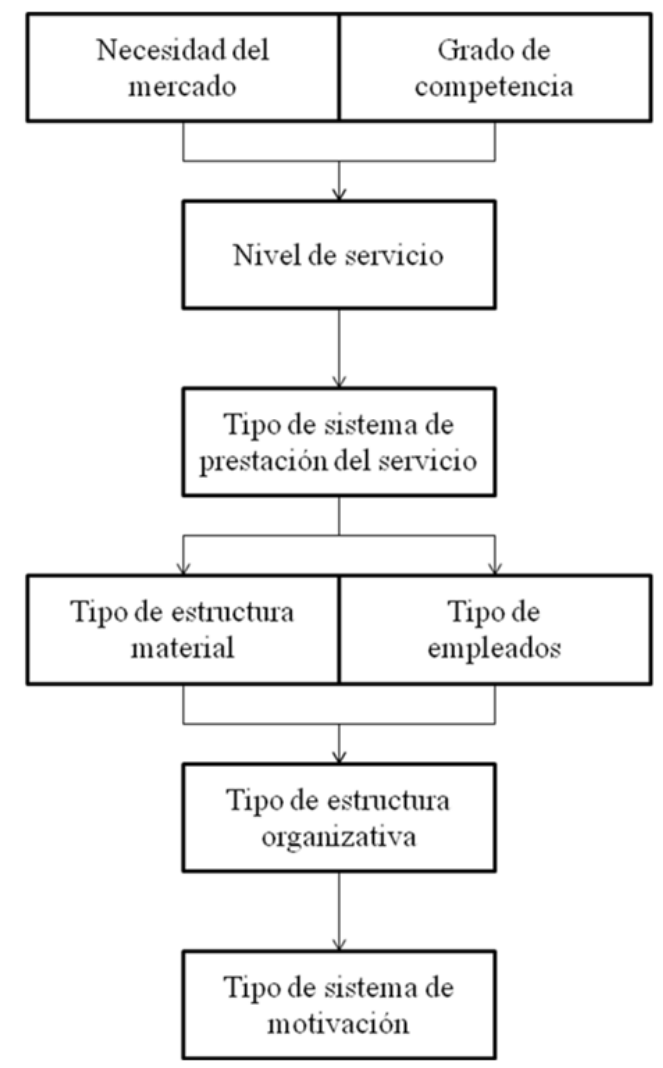

Figura 1 - Plan de organización

Fuente: Sasser et al.(1978) citado en Flipo (1993), p. 129

Toda la literatura relativa a la gestión de empresas de servicios se muestra acorde con el plan propuesto por Sasser et al. (1978). No obstante, esta postura, teniendo en cuenta los poderes reales no los formales, sugiere que se debe invertir la estructura habitual de los organigramas, situando en la cúspide el mercado y el personal en contacto.

En este sentido, Davidson (1978) escribe un artículo que titula: “Como tener éxito en una industria de servicios... poned al revés el organigrama", donde remarca la importancia de esta posturaque comenzó a esbozarse cuando el nivel de competencia no era tan elevado como el actual.

\subsection{Gestión del Soporte Físico}

El soporte físico, como ya es sabido, es uno de los elementos del sistema de producción de servicios. Se trata de un soporte material del que se servirán el personal en contacto y/o los clientes y que resulta necesario para que se produzca el servicio. Este soporte puede dividirse en dos categorías: 
- Los instrumentos necesarios para el servicio: Todos los objetos tanto las máquinas como los muebles puestos a disposición del personal en contacto y/o clientes forman parte de esta categoría. La utilización de estos elementos permite la realización del servicio.

- El entorno: Todo lo que rodea a los instrumentos mencionados en el punto anterior conforman esta categoría. Se trata fundamentalmente de la localización, los edificios, el decorado y la disposición en la que se efectúa la producción del servicio.

Por tanto, aunque un servicio no deja de ser un bien intangible, pudiendo describirlo como un simple acto, el soporte físico -equipo e instalaciones- puede desempeñar un papel muy importante en la producción del mismo, tal como el que se le asigna en el sector manufacturero. Son numerosas las empresas que emplean grandes medios en este cometido, sin que ello necesariamente anule o ensombrezca la importancia de otros elementos. De hecho, cuanto más equipo o capital se utilice, mayores serán las probabilidades de que el uso eficaz de los recursos físicos dependa de la capacidad para dirigir los esfuerzos del personal en contacto y la participación de los clientes (Norman, 1990). Con la incorporación de las nuevas tecnologías, la presencia del personal en contacto puede verse reducida, incrementando por el contrario la participación del cliente.

No obstante, son pocos los investigadores que han estudiado las interacciones entre el consumidor y los medios tecnológicos (véase tabla 2), y de ellos la mayoría se centra en un caso concreto con una tecnología sencilla. Muy pocos son los que intentan examinar el conjunto de las tecnologías self-service de las que se dispone en la actualidad. En este sentido, Meuter, Ostrom, Roundtree y Bitner (2000) en su estudio incorporan diversas tecnologías self-service -unas más establecidas que otras-, aunque trabajan con ellas de forma conjunta, es decir, sin tratar de realizar comparaciones (véase tabla 3).

Tabla 2

Principales referencias bibliográficas

\begin{tabular}{|c|c|c|}
\hline \multirow{3}{*}{$\begin{array}{l}\text { Interacciones entre el } \\
\text { consumidor y la } \\
\text { tecnología }\end{array}$} & Desarrollo de perfiles de usuarios & $\begin{array}{l}\text { Langeard, Bateson, Lovelock y Eiglier (1981); Bateson } \\
\text { (1985); Zeithaml y Gilly (1987); Darian (1987); Greco y } \\
\text { Fields (1991); Eastlick (1996). }\end{array}$ \\
\hline & Actitudes del consumidor & $\begin{array}{l}\text { Raub (1981); Dabholkar (1992); Dabholkar (1996a); } \\
\text { Parasuraman (1998); Bobbitt y Dabholkar (2001); } \\
\text { Dabholkar y Bagozzi (2002); Walker, Craig-Lees, Hecker } \\
\text { y Francis (2002); Dabholkar, Bobbitt y Lee (2003); } \\
\text { Meuter, Ostrom, Bitner y Roundtree (2003); Snellman y } \\
\text { Vihtkai (2003); Meuter, Bitner, Ostrom y Brown (2005); } \\
\text { Proença y Rodrigues (2011). }\end{array}$ \\
\hline & $\begin{array}{l}\text { Evolución de la satisfacción del } \\
\text { cliente }\end{array}$ & $\begin{array}{l}\text { Meuter et al. (2000); Anselsson (2001); Agnihothri, } \\
\text { Sivasubramaniam y Simmons (2002); Walker et al. } \\
\text { (2002); Beatson, Lee y Coote (2007). }\end{array}$ \\
\hline
\end{tabular}

Nota. Fuente: Elaboración propia

Categorías de tecnologías self-service en uso

TECNOLOGIA

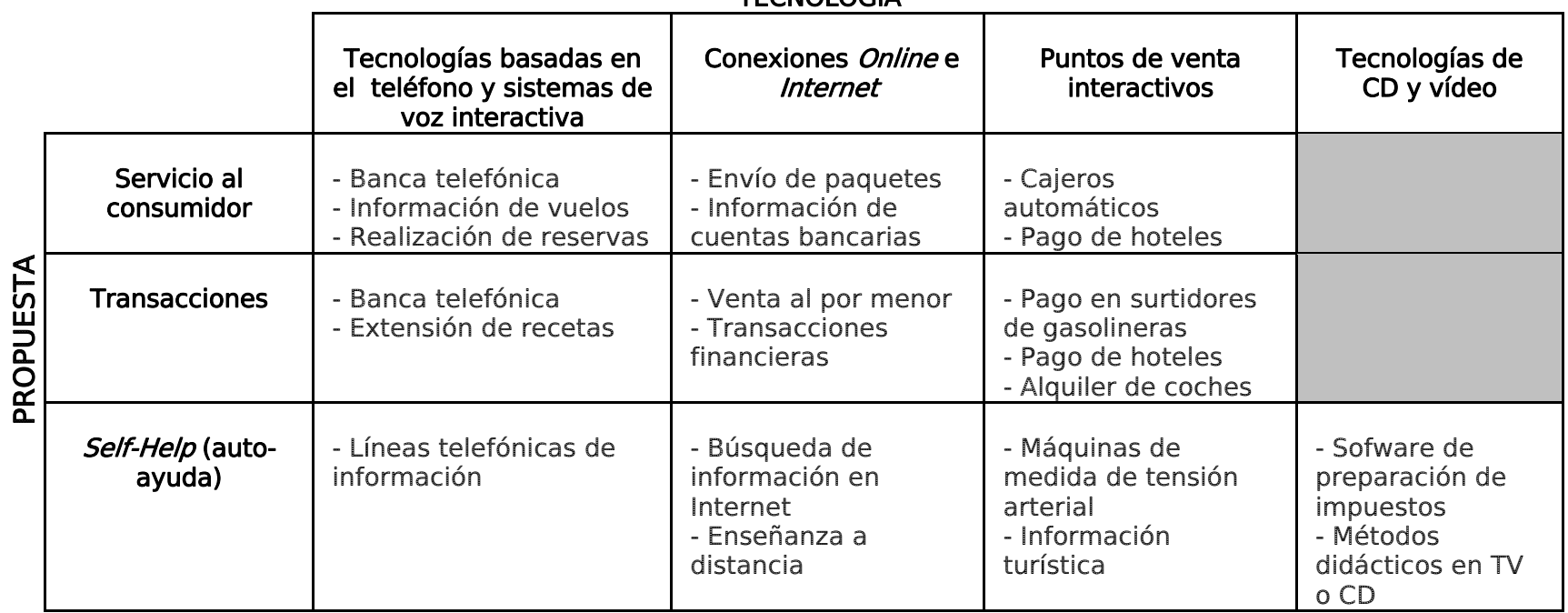

Nota. Fuente: Meuter, M.L. et al.. (2000), p. 52 
En la tabla 3 se presentan distintos ejemplos de tecnologías self -service. Las columnas de la matriz representan los tipos de tecnologías que las empresas usan con los consumidores como medio en los encuentros self-service: tecnologías basadas en el teléfono y sistemas de voz interactiva, conexiones Online e Internet, quioscos interactivos gratuitos, y tecnologías de CD y vídeo. Las filas representan las propuestas de tecnología desde la perspectiva del consumidor, es decir, lo que el consumidor puede llevar a cabo por medio del uso de la tecnología: servicio al consumidor, transacciones ${ }^{3}$-la tecnología permite a los usuarios encargar, comprar e intercambiar recursos con empresas sin ninguna interacción directa con sus empleados-, y Self-Help -se refiere a tecnologías que permiten a los consumidores aprender, recibir información, entrenarse por sí mismos y disponer de sus propios servicios-.

\section{Service Encounter y Automatización: Análisis Empírico}

Una vez estudiados los elementos que intervienen en la prestación del servicio se ha analizado cómo la incorporación de procesos automatizados provoca cambios en dichos elementos. Para ello se ha realizado un estudio empírico sobre cuatro subsectores ${ }^{4}$ : autopistas, aparcamientos, lavado de automóviles y alquiler de películas cinematográficas; centrando la atención en aquellos procesos de automatización que afectan a la relación cliente-empresa, lo que se ha dado en llamar automatización externa.

\subsection{Obtención de Datos}

Dentro de estos sectores se ha trabajado sobre la población conformada por aquellas empresas ubicadas en territorio español, con un nivel de facturación superior o igual a 300.000 euros en el año 2000. El tamaño de la población es de 202 empresas $^{5}$, de las cuales 10 pertenecen al sector de las autopistas (4,95\%), 112 al de aparcamientos (55,44\%), 49 al de lavado de automóviles $(24,26 \%)$ y 31 al de alquiler de películas cinematográficas $(15,35 \%)$.

De las 202 empresas que conforman la población, han sido encuestadas 99, seleccionadas a través de un muestreo aleatorio estratificado con un error muestral del $7 \%$ y un nivel de confianza del $95 \%$. El procedimiento de recogida de la información ha sido a través de una encuesta postal o telefónica entre Marzo y Junio de 2001.

De las 99 empresas que conforman la muestra, 7 pertenecen al sector de las autopistas, 48 al de aparcamientos, 26 al de lavado de automóviles y 18 al de alquiler de películas cinematográficas (véase figura 2). De todas ellas, 23 manifiestan no haber vivido, en los últimos años, un proceso de automatización que haya afectado a su relación con el cliente, siendo el motivo principal -según el criterio de las propias empresas- el hecho de que se encuentren suficientemente automatizadas, siguiendo en importancia el hecho de que el cliente no lo demande. Las 76 empresas restantes manifiestan haber vivido, en los últimos años, algún proceso de automatización que haya afectado a su relación con el cliente, siendo la causa que toma mayor importancia la relativa a la aparición de nuevas tecnologías, seguida del ritmo impuesto por los competidores, las exigencias de los clientes y, finalmente, otras causas que carecen de relevancia. Sobre este último grupo es, precisamente, donde se ha centrado el análisis.

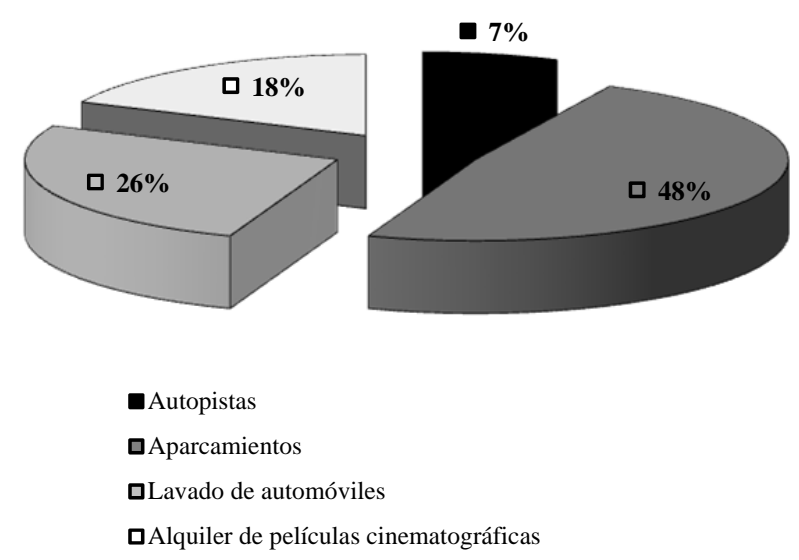

Figura 2 - Distribución de la Muestra por Subsectores

Fuente: Elaboración propia 


\subsection{Definición de las variables}

En este análisis se ha trabajado con una serie de variables que tratan de recoger las posibles variaciones sufridas por los elementos pertenecientes al service encounter ante la incorporación de procesos automatizados. Estas variables se presentan en la tabla 4.

Tabla 4

Descripción de las Variables

\begin{tabular}{|c|c|c|}
\hline Elemento & Variables & Tipo de variable \\
\hline Servicio & Evolución del número de servicios a ofrecer & Variable métrica \\
\hline \multirow{2}{*}{ Personal en contacto } & $\begin{array}{l}\text { Evolución del número de empleados que forman parte del } \\
\text { personal en contacto }\end{array}$ & Variable métrica \\
\hline & \multirow[t]{2}{*}{$\begin{array}{l}\text { Evolución del grado de relación entre el personal en contacto } \\
\text { y el cliente }\end{array}$} & \multirow[t]{2}{*}{ Variable categórica } \\
\hline \multirow{2}{*}{ Cliente } & & \\
\hline & Evolución del grado de participación del cliente & Variable categórica \\
\hline Otros clientes & Evolución del grado de relación entre los clientes & Variable categórica \\
\hline Soporte físico & \multicolumn{2}{|c|}{$\begin{array}{l}\text { Por lo que respecta al soporte físico se analizan las últimas incorporaciones tecnológicas } \\
\text { realizadas que permitirán medir el grado de automatización }\end{array}$} \\
\hline \multirow[t]{4}{*}{$\begin{array}{l}\text { Sistema de organización } \\
\text { interna }\end{array}$} & $\begin{array}{l}\text { Evolución de la capacidad del personal para tomar } \\
\text { decisiones (grado de centralización-descentralización) }\end{array}$ & Variable categórica \\
\hline & $\begin{array}{l}\text { Evolución del número de normas y procedimientos } \\
\text { estandarizados que guían la actuación de los empleados } \\
\text { (grado de formalización) }\end{array}$ & Variable categórica \\
\hline & $\begin{array}{l}\text { Evolución del número de categorías laborales (grado de } \\
\text { especialización vertical) }\end{array}$ & Variable categórica \\
\hline & $\begin{array}{l}\text { Evolución del nivel de especialización de los trabajadores } \\
\text { (nivel de especialización horizontal) }\end{array}$ & Variable categórica \\
\hline
\end{tabular}

Nota. En las variables categóricas se utiliza una Escala tipo Likert de cinco puntos, donce el 1 sigunifica se ha reducido mucho y el 5 se ha incrementado mucho.

Fuente: Elaboración propia

Finalmente para recoger el grado de automatización se ha trabajado con la variable categórica "gautom", analizando una serie de aspectos relativos a cada sector, establecidos tras conversaciones mantenidas con proveedores de tecnología y con empresas propias de los distintos subsectores. En el sector autopistas se ha centrado la atención en el pago de peajes, estableciendo el grado de automatización en función del porcentaje de carriles automatizados sobre el total, teniendo en cuenta el tipo de automatización utilizada -telepeaje, tarjeta magnética, máquina contadora de dinero-. En el caso de los aparcamientos se ha analizado si disponen de sistema automatizado de pago, señal informativa de número de plazas libres, sistemas de detección de plazas libres, sistema guiado de aparcamiento, sistema de colocación automática del automóvil y si estos medios tecnológicos están disponibles para todas las plazas de aparcamiento o para una parte (no habiendo grandes diferencias en este aspecto). Para el lavado de automóviles se ha centrado la atención en los sistemas automáticos con cepillos -tipo puente o tipo tunel-, se ha analizado si estaban totalmemte automatizados o semiautomatizados (fácil de distinguir a través del modelo utilizado), se ha tenido en cuenta el número de equipos complementarios utilizados máquinas cobradoras, aspiradoras, sistema de dispensarios, máquina lava tapetes- y si el sistema automatizado se utiliza en todos los casos o si se dispone de otros sistemas. En el sector alquiler de películas cinematográficas se ha trabajado con el porcentaje que representan el número medio de cintas de video y DVDs a disposición del cliente en cajeros sobre el total ofertado, sin resultar demasiado significativas las diferencias tecnológicas entre los distintos cajeros ${ }^{6}$.

\subsection{Resultados}

Se ha empleado un ANOVA (análisis de la varianza) para contrastar las hipótesis nulas en las que en categorías distintas de la variable "gautom" (variable independiente), las variables "evolución del número de servicios a ofrecer" y "evolución del número de empleados que forman parte del personal en contacto" (variables métricas dependientes) presentan cada una de ellas las mismas medias ${ }^{7}$. En la tabla 5 se recogen los resultados de este análisis.

Por otro lado, se analizó, mediante las medidas Chi-cuadrado de Perason, Gamma, Tau-c de Kendall y D de Somer, la existencia o no de asociación entre la variable "gautom" y el resto de variables todavía no estudiadas. Se han elegido estas medidas por tratarse de variables ordinales. Estas herramientas permiten 
probar la hipótesis nula de independencia en cada uno de los cruces entre las variables citadas. Tanto el estadístico Gamma, como el coeficiente Tau-b de Kendall y el D de Somer toman valores entre -1 y +1 , alcanzando el valor 0 si son independientes. Los resultados de este análisis se presentan en la tabla 6.

Tabla 5

ANOVA

\begin{tabular}{|l|c|}
\cline { 2 - 2 } \multicolumn{1}{l}{ VARIABLES DEPENDIENTES } & VARIABLE INDEPENDIENTE \\
\cline { 2 - 2 } & Grado de automatización \\
\hline $\begin{array}{l}\text { Evolución del número de servicios a ofrecer } \\
\text { contación del número de empleados que forman parte del personal en }\end{array}$ & 0,250 \\
\hline
\end{tabular}

Nota: Los valores presentados indican el nivel de significación

Fuente: Elaboración propia

Tabla 6

Resultados de las medidas Chi-cuadrado de Pearson, Gamma, Tau-c de Kendall y D de Somer

\begin{tabular}{|c|c|c|c|}
\hline & \multicolumn{3}{|c|}{ Grado de automatización } \\
\hline $\begin{array}{l}\text { Evolución del grado de relación entre el } \\
\text { personal en contacto y el cliente }\end{array}$ & 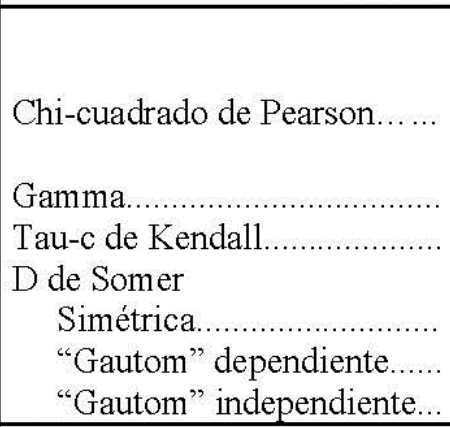 & $\begin{array}{c}\text { Valor } \\
98,452 \\
(\mathrm{GL}: 12) \\
-0.798 \\
-0.469 \\
\\
-0.557 \\
-0.525 \\
-0.593 \\
\end{array}$ & $\begin{array}{c}\text { Significación } \\
\text { aproximada } \\
0.000 \\
0.000 \\
0.000 \\
\\
0.000 \\
0.000 \\
0.000\end{array}$ \\
\hline $\begin{array}{l}\text { Evolución del grado de participación del } \\
\text { cliente }\end{array}$ & $\begin{array}{l}\text { Chi-cuadrado de Pearson....... } \\
\text { Gamma............................... } \\
\text { Tau-c de Kendall.................. } \\
\text { D de Somer } \\
\quad \text { Simétrica...................... } \\
\text { "Gautom" dependiente...... } \\
\text { "Gautom" independiente... }\end{array}$ & $\begin{array}{c}\text { Valor } \\
\\
146,814 \\
(\mathrm{GL}: 8) \\
0.946 \\
0.731 \\
\\
0.777 \\
0.732 \\
0.827 \\
\end{array}$ & $\begin{array}{c}\text { Significación } \\
\text { aproximada } \\
0.000 \\
0.000 \\
0.000 \\
0.000 \\
0.000 \\
0.000\end{array}$ \\
\hline $\begin{array}{l}\text { Evolución del grado de relación entre los } \\
\text { clientes }\end{array}$ & $\begin{array}{l}\text { Chi-cuadrado de Pearson....... } \\
\text { Gamma................................. } \\
\text { Tau-c de Kendall................... } \\
\text { D de Somer } \\
\quad \text { Simétrica......................... } \\
\text { "Gautom" dependiente....... } \\
\text { "Gautom" independiente... }\end{array}$ & $\begin{array}{c}\text { Valor } \\
88,546 \\
(G L: 12) \\
-0.759 \\
-0.484 \\
-0.562 \\
-0.530 \\
-0.599\end{array}$ & $\begin{array}{c}\text { Significación } \\
\text { aproximada } \\
0.000 \\
0.000 \\
0.000 \\
0.000 \\
0.000 \\
0.000\end{array}$ \\
\hline $\begin{array}{l}\text { Evolución del grado de } \\
\text { centralización/descentralización }\end{array}$ & $\begin{array}{l}\text { Chi-cuadrado de Pearson....... } \\
\text { Gamma............................... } \\
\text { Tau-c de Kendall................... } \\
\text { D de Somer } \\
\text { Simétrica........................ } \\
\text { "Gautom" dependiente...... } \\
\text { "Gautom" independiente... }\end{array}$ & $\begin{array}{c}\text { Valor } \\
14,527 \\
(\mathrm{GL}: 8) \\
0.104 \\
0.044 \\
\\
0.061 \\
0.064 \\
0.059\end{array}$ & $\begin{array}{c}\text { Significación } \\
\text { aproximada } \\
0,268 \\
0.565 \\
0.565 \\
0.565 \\
0.565 \\
0.565\end{array}$ \\
\hline
\end{tabular}

Continúa... 
Continuación...

\begin{tabular}{|c|c|c|c|}
\hline Evolución del grado de formalización & $\begin{array}{l}\text { Chi-cuadrado de Pearson...... } \\
\text { Gamma........................... } \\
\text { Tau-c de Kendall ................. } \\
\text { D de Somer } \\
\text { Simétrica..................... } \\
\text { "Gautom" dependiente...... } \\
\text { "Gautom" independiente... }\end{array}$ & $\begin{array}{c}\text { Valor } \\
3,330 \\
(\mathrm{GL}: 8) \\
0.167 \\
0.036 \\
\\
0.059 \\
0.090 \\
0.044\end{array}$ & $\begin{array}{l}\text { Significación } \\
\text { aproximada } \\
0,766 \\
0.519 \\
0.519 \\
0.519 \\
0.519 \\
0.519\end{array}$ \\
\hline $\begin{array}{l}\text { Evolución del grado de especialización } \\
\text { vertical }\end{array}$ & \begin{tabular}{|l} 
Chi-cuadrado de Pearson....... \\
Gamma.............................. \\
Tau-c de Kendall................... \\
D de Somer \\
Simétrica....................... \\
"Gautom" dependiente...... \\
"Gautom" independiente...
\end{tabular} & $\begin{array}{l}\text { Valor } \\
28,821 \\
(\mathrm{GL}: 8) \\
-0,152 \\
-0.074 \\
-0.094 \\
-0.088 \\
-0.101 \\
\end{array}$ & $\begin{array}{c}\text { Significación } \\
\text { aproximada } \\
0,010 \\
\\
0.364 \\
0.364 \\
\\
0.364 \\
0.364 \\
0.364 \\
\end{array}$ \\
\hline $\begin{array}{l}\text { Evolución del grado de especialización } \\
\text { horizontal }\end{array}$ & $\begin{array}{l}\text { Chi-cuadrado de Pearson....... } \\
\text { Gamma................................. } \\
\text { Tau-c de Kendall................... } \\
\text { D de Somer } \\
\quad \text { Simétrica.......................... } \\
\text { "Gautom" dependiente...... } \\
\text { "Gautom" independiente... }\end{array}$ & $\begin{array}{c}\text { Valor } \\
\\
15,335 \\
(\mathrm{GL}: 12) \\
0.175 \\
0.048 \\
\\
0.079 \\
0.097 \\
0.066\end{array}$ & $\begin{array}{l}\text { Significación } \\
\text { aproximada } \\
0,082 \\
0.485 \\
0.485 \\
\\
0.485 \\
0.485 \\
0.485\end{array}$ \\
\hline
\end{tabular}

Nota. El estadístico Chi-cuadrado de Pearson debe ser interpretado con cierta cautela, puesto que en la mayoría de las tablas de contingencia, el porcentaje de casillas con frecuencias esperadas menores que 5 es superior al $20 \%$. Fuente: Elaboración propia

\subsection{Análisis de los resultados}

Estos resultados muestran que se puede establecer una asociación entre el grado de automatización y la evolución del número de empleados que forman parte del personal en contacto, puesto que se ha rechazado la hipótesis nula en la que la media de los valores de la variable "evolución del número de empleados que forman parte del personal en contacto" no varía ante distintos valores de la variable "gautom".

También puede hablarse de una relación entre el grado de automatización y la evolución del grado de relación entre el personal en contacto y el cliente, la evolución del grado de participación del cliente y la evolución del grado de relación entre los clientes; al rechazarse las hipótesis nulas relativas a la independencia entre estos pares de variables. Se manifiesta una asociación media e inversa entre el grado de automatización y la evolución del grado de relación entre el personal en contacto y el cliente pero significativa a cualquier nivel, donde el grado de automatización podría actuar como variable independiente. En la relación entre el grado de automatización y la evolución del grado de participación del cliente la asociación tiende a fuerte y es directa, pudiendo actuar la primera como independiente. Esto mismo ocurre en la relación entre el grado de automatización y la evolución del grado de relación entre los clientes donde la asociación no es tan fuerte e inversa, pero igualmente significativa a cualquier nivel.

En definitiva, los datos ponen de manifiesto que cuanto mayor es el grado de automatización, menor es el número de empleados que forman parte del personal en contacto, mayor es la participación del cliente, y menor la relación entre los clientes y de éstos con el personal en contacto.

Sin embargo, no se manifiesta una relación entre el grado de automatización y la evolución del número de servicios a ofrecer. Ante la incorporación de procesos automatizados, el número de servicios a ofrecer por la empresa se mantiene estable en la mayoría de los casos estudiados (74\%), se incrementa en el $14 \%$ 
de los mismos y disminuye en el $12 \%$ de las unidades encuestadas. Pudiendo detectar que la disminución del número de servicios suele ir acompañada de la eliminación del sistema productivo utilizado antes de la automatización (véase figura 3).

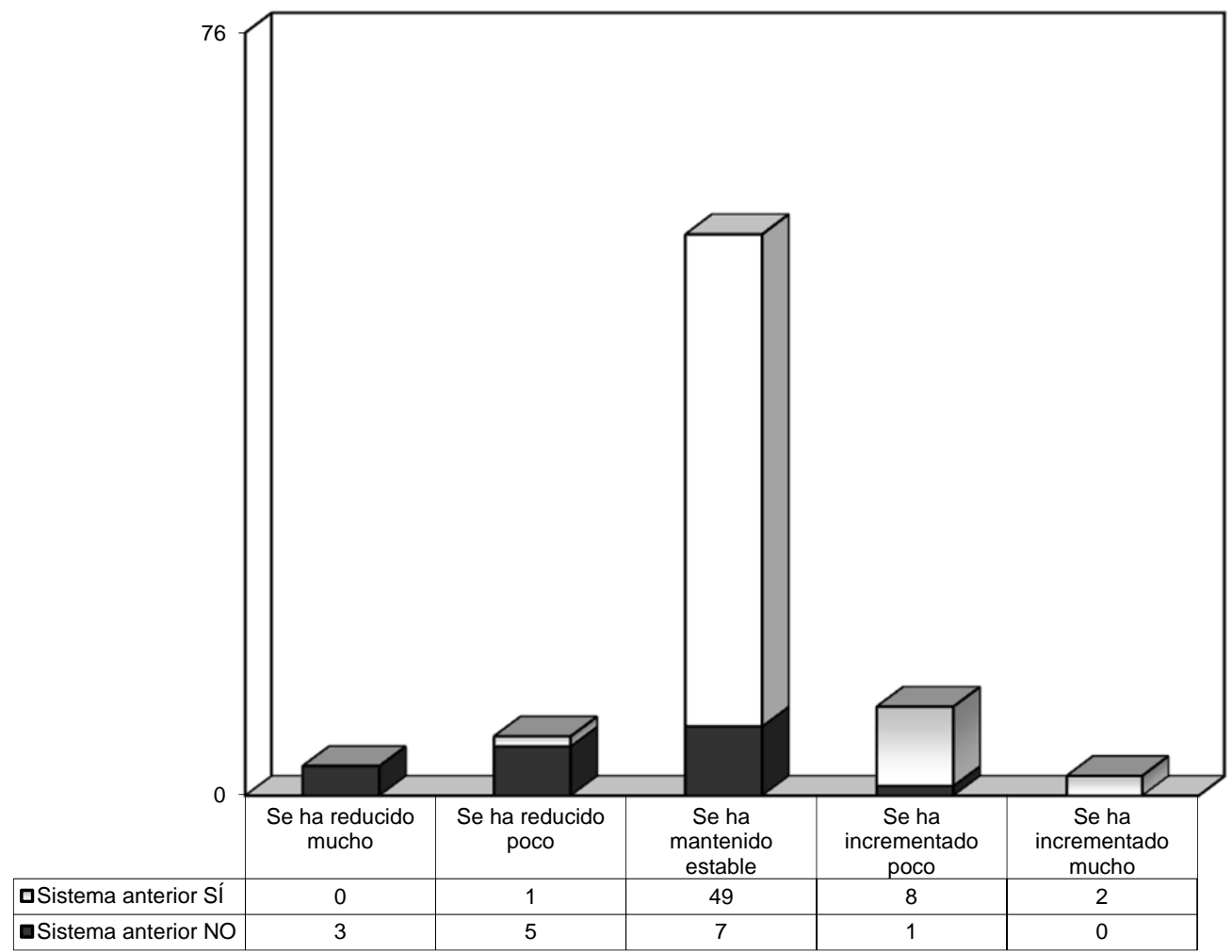

Figura 3 - Evolución del Número de Servicios a Ofrecer Fuente: Elaboración propia

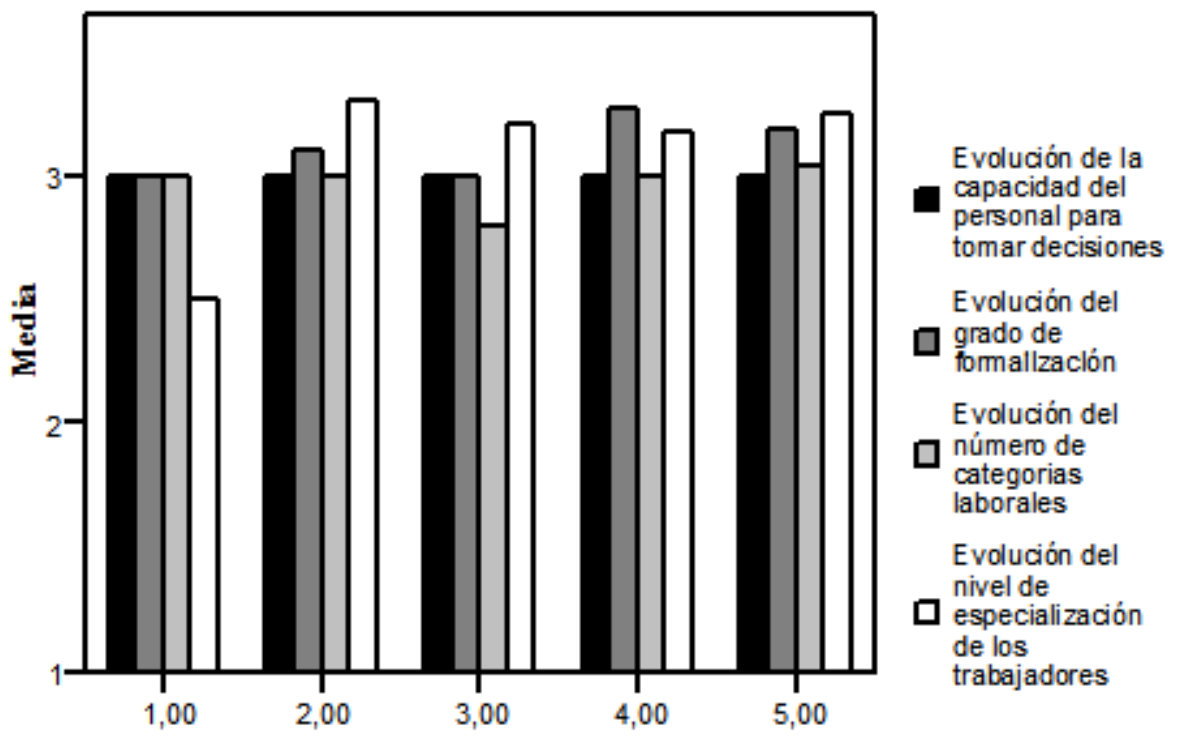

Grado de automatización

Eje Y: 1: Se ha reducido mucho; 2: Se ha reducido poco; 3: Se ha mantenido estable; 4: Se ha incrementado poco; 5: Se ha incrementado mucho

Figura 4. Evolución del sistema de organización interna

Nota. Aunque no corresponde el cálculo de la media para las variables representadas en el eje X, se ha obtenido con el objetivo de mostrar la falta de evolución de las mismas ante la incorporación de procesos automatizados, tal como ya se acaba de indicar. Dentro del nivel de automatización "1", solamente se han posicionado dos empresas, por lo que las barras correspondientes a este valor no se pueden considerar representativas.

Fuente: Elaboración propia 
Teniendo en cuenta las medidas de asociación Gamma, Tau-c de Kendall y D de Somer, tampoco se constata una relación entre el grado de automatización y las variables relativas al sistema de organización interna -grado de centralización/descentralización, grado de formalización, grado de especialización vertical y grado de especialización horizontal-, situación influenciada posiblemente por la elección de la población objeto de estudio. Estas variables han permanecido estables en la mayoría de los casos ante cualquier nivel de automatización, el grado de centralización/descentralización se mantiene constante para 74 de 76 empresas encuestadas, el nivel de formalización para 64 de las 76, el grado de especialización vertical para 73 y el nivel de especialización horizontal para 58. Respecto al nivel de formalización, de las 12 restantes, 11 de ellas manifiestan un pequeño incremento ante la automatización. En el caso del nivel de especialización horizontal, de las 18 restantes, 16 también manifiestan un pequeño incremento (véase figura 4). No obstante, el estadístico Chi-cuadrado de Pearson pone de manifiesto una relación entre las variables "Grado de automatización" y "Evolución del grado de especialización vertical" que debe ser interpretada con mucha cautela, puesto que en la tabla de contingencia el porcentaje de casillas con una frecuencia menor que 5 es del $80 \%$ (12 de 15 casillas).

\section{Conclusiones}

Para la prestación de un bien intangible es necesaria la interacción entre la empresa prestataria del mismo y el cliente. La primera se relaciona con el segundo a través del personal en contacto y/o del soporte físico, no necesitándose la presencia de ambos elementos para que la producción del servicio tenga lugar.

La incorporación de procesos automatizados supone ciertas modificaciones en los elementos que intervienen en el service encounter, influyendo en la forma en que el proveedor del servicio se relaciona con el consumidor del mismo. El objetivo de este trabajo es analizar cómo evolucionan estos elementos en los subsectores: autopistas, aparcamientos, lavado de automóviles y alquiler de películas cinematográficas.

En estos sectores cuanto mayor es el nivel de automatización mayor suele ser la presencia del soporte físico en detrimento del personal en contacto. La disminución del número de empleados que conforman el personal en contacto tiene lugar gracias a una mayor implicación por parte del cliente, lo que podría suponer importantes ahorros en costes, y como consecuencia, mejoras en productividad siempre que se mantengan los niveles de calidad. No obstante, esta menor presencia del personal en contacto también podría repercutir negativamente sobre otra prioridad competitiva, el servicio al cliente, debido a una menor atención personalizada. Pero una menor relación entre el personal en contacto y el cliente, a la que le podríamos unir una menor relación entre los clientes, permite disponer de un mayor grado de intimidad, aspecto valorado positivamente por el cliente, al que se le podría unir mejoras en tiempo y un mayor grado de accesibilidad al servicio. Todo ello, teniendo en cuenta que el número de servicios a ofrecer se mantiene estable en la mayoría de las empresas estudiadas, y que en el resto de los casos se incrementa en un porcentaje de empresas mayor al porcentaje de empresas en el que disminuye. Esto permite esperar mejoras ya no solamente en el nivel de servicio al cliente sino también en el grado de flexibilidad.

Por otra parte, ante la incorporación de procesos automatizados no se han constatado grandes alteraciones a nivel organizativo, simplemente incrementos puntuales del nivel de formalización y especialización horizontal. Cambios que no tendrían por qué tener efectos negativos sobre los distintos objetivos del subsistema de operaciones.

En definitiva, los resultados ponen de manifiesto que la incorporación de procesos de automatización junto con los cambios en los elementes del subsistema de operaciones que esto provoca, podría tener efectos positivos sobre las prioridades competitivas sin la presencia de trade offs entre las mismas. En concreto, los cambios experimentados por los elementos participantes en el service encounter auguran cotas aceptables de productividad y flexibilidad, variables tradicionalmente antagónicas.

Esta situación, demanda pues nuevos estudios que, por un lado, analicen la incidencia que la incorporación de procesos automatizados podría tener sobre los objetivos del subsistema de operaciones y que, por otro, puedan hacer extensibles estas conclusiones a otros subsectores, cuestiones que están siendo abordadas por las autoras en nuevos trabajos. 


\section{Notas}

1- Para un mayor conocimiento sobre la relación entre el service encounter y el valor del servicio vease: Gil, Sánchez, Berenguer y González (2005)..

2- Diversos autores han preferido considerar el proceso de entrega del servicio no como una interacción puntual, sino como una cascada de encuentros o interacciones (Liljander y Strandvik, 1995; Gil et al., 2005), en cada uno de los cuales pueden participar distintos elementos.

3- Hof en 1999, al igual que otros autores, pone de manifiesto el rápido crecimiento de las transacciones en Internet para ventas a consumidores y entre empresas.

4- Códigos SIC:

4784: Autopistas, puentes y túneles de peaje

7523: Aparcamientos al aire libre

7525: Aparcamientos en edificios y locales subterráneos

7542: Lavado de automóviles

7823: Alquiler de películas cinematográficas

5- Se ha utilizado la base de datos D\&b Marketing 2000/3 (Dun \& Bradstreet), la cual ofrecía una población conformada por 237 empresas, pero tras una llamada telefónica inicial para contrastar la existencia de las mismas se detectan ciertas fusiones, cierres, etc.; lo que reduce la población a 202 entidades.

6- Se han establecido intervalos para definir las categorías.

7- En el análisis de la varianza solamente se ha trabajado con dos variables dependientes por ser éstas variables métricas, siendo las restantes variables categóricas, razón por la que no procede dicho análisis

\section{Bibliografía}

Agnihothri, S., Sivasubramaniam, N., \& Simmons, D. (2002). Levering Technology to Improve Field Service. International Journal in Service Industry Management, 13(1), 47-68. http://dx.doi.org/10.1108/09564230210421155

Anselmsson, J. (2001). Customer-perceived Service Quality and Technology-based Self-service. Doctoral dissertation, Lund, Lund University: Lund Business Press.

Ba, S., Stallaert, J., \& Zhang, Z. (2010). Balancing IT with the Human Touch: Optimal Investment in IT-Based Customer Service. Info. Sys. Research, 21(3), September, 423-442.

http://dx.doi.org/10.1287/isre.1100.0282

Bateson, J. (1985a). Perceived Control and the Service Encounter. En Czpiel, John A., Solomon, Michael R., \& Surprenant, Carol F. (Eds.). The Service Encounter. MA, Lexington: Lexington Books.

Bateson, J. E. (1985b). Sef Service Consumer. An Exploratury Study. Journal of Retailing, 61(3), 49-76.

Beatson, A., Lee, N., \& Coote, L. (2007). Self-Service Technology and the Service Encuounter. Service Industries Journal, 27(1), 75-89. http://dx.doi.org/10.1080/02642060601038700

Bernillón, A., \& Cerutti, O. (1989). Implantar y Gestionar la Calidad Total. Barcelona: Ediciones Gestión 2000.

Berry, L. L. (1981). The Employee as Customer. Journal of Retail Banking, 3(1).

Bettencourt, L., \& Gwinner, K. (1996). Customization of the Service Experience: The Role of the Frontline Employee. International Journal in Service Industry Management, 7(2), 2-20.

http://dx.doi.org/10.1108/09564239610113442

Bianco, A. (1987, agosto). The Decline of the Superstar. Busines Week.

Bitner, M. J., Booms, B. H., \& Tetreault, M. S. (1990). The Service Encounter: Diagnosing Favorable and Unfavorable Incidents. Journal of Marketing, 54, Janaury, 71-84.

Bitner, M. J., Brown, S. W., \& Meuter, M. L. (2000). Technology Infusion in Service Encounters. Journal of the Academy of Marketing Science, 28(1), 138-149. http://dx.doi.org/10.1177/0092070300281013

Bitner, M. J., Faranda, W. T., Hubebert, A. R., \& Zeithaml, V. A. (1997). Customer Contributions and Roles in Service Delivery. International Journal of Service Industry Management, 8(3), 193-205.

http://dx.doi.org/10.1108/09564239710185398 
Bobitt, L. Michele, \& Dabholkar, Pratibha A. (2001). Integrating Attitudinal Theories to Understand and Predict Use of Technology-Based Self-Service: The Internet as an Illustration. International journal of Service Industry Management, 12(5), 423-450. http://dx.doi.org/10.1108/EUM0000000006092

Bonoma, T. V. (1987). Los Subsectores del Marketing. Harvard-Deusto Business Review, 3을 trimestre. Bowden, B. (2002). Customers Help Check out New Technology. Arkansas Business, 19(5), 15-18.

Bowen, D. E., \& Schneider, B. (1985). Boundary Spanning Role Employees and the Service Encounter: Some Guidelines for Management Research. eEn Czpiel, J.A., Solomon, M.R., \& Surprenant, C.F. (Eds.). The Service Encounter. MA, Lexington: Lexington Books.

Carlzon, J. (1988). El Momento de la Verdad. Bilbao: Asociación para el Progreso de la Dirección. Chase, R. B. (1985). The Ten Commandments of Service System Management. Interfaces, 15(3). http://dx.doi.org/10.1287/inte.15.3.68

Chase, R. B. (1987). Where does the Costomer Fit in a Service Operation?. Harvard Business Review, 56(4), Noviembre-Diciembre, 137-142.

Chase, R. B. (1992). The Customer Contact Approach to Services: Theoretical Bases and Practical Extensions. En Lovelock, C.H. (Ed.) Managing Services. Marketing, Operations, and Human Resources (pp. 43-49). USA: Prentice-Hall,.

Chase, R. B., \& Aquilano, N. J. (1994). Dirección y Administración de la Producción y de las Operaciones. Argentina: Addison-Wesley.

Clemmer, E. C., \& Schneider, B. (1996). Fair Service. En Swartz, Teresa A., Bowen, David E., \& Brown, Stephen W. (Eds.). Advances in Services Marketing and Management (vol. 5, pp. 109-126). CT, Greenwich: JAl Press.

Czepiel, J. A. (1990). Relationships with Customer: A Differentiating Philosophy of Marketing". En Bowen, D.E., Chase, R. B., Cummings, T. G., \& Asociados. Service Management Effectiveness. Balansing Strategy, Organization and Human Resources, Operations, and Marketing (pp. 299-323). USA: Jossey-Bass Publishers,.

Czepiel, J. A., Solomon, M. R., \& Surprenant, C. F. (1985). The Service Encounter. Lexington Books.

Dabholkar, P. A. (1992). Role of Affect and Need for Interaction in On-Site Service Encounters. En Sherry, J.F., \& Sternthal, B. (Eds.). Advances in Consumer Research (vol. 19, pp. 563-569). UT, Provo: Association for Consumer Research.

Dabholkar, P. A. (1996a). Consumer Evaluations of New Technology-Based Self-Service Options: An Investigation of Alternative Models of Service Quality. International Journal of Research in Marketing, 13(1), 29-51. http://dx.doi.org/10.1016/0167-8116(95)00027-5

Dabholkar, P. A. (1996b). Technology-Based Service Delivery: A Classification Scheme for Developing Marketing Strategies. En Swartz, T.A., Bowen, D.E., \& Brown, S.W. (Eds.). Advances in Services Marketing and Management (vol. 3, pp. 241-271). CT, Greenwich: JAl Press

Dabholkar, P. A., \& Bagozzi, R. P. (2002). An Attitudinal Model of Technology-Based Sef-Service: Moderating Effects of Consumer Traits and Situational Factors. Journal of the Academy of Marketing Science, 30(3), 184-201.

Dabholkar, P. A., Bobbitt, L. M., \& Lee, E. (2003). Understanding Consumer Motivation and Behavior Related to Self-Scanning in Retailing. International Journal of Service Industry Management, 14(1), $59-95$. http://dx.doi.org/10.1108/09564230310465994

Darian, J. C. (1987). In-Home Shopping: Are There Consumer Segments?. Journal of Retailing, 63(2), 163186.

Davidson, D. S. (1978). How to Succed in a Service Industry... Turn the Organization Chart Upside Down. Management Review, Abril, 13-16.

Diz, M. E. (2005). Subsistema de Operaciones en Empresas de Servicios: Una Reflexión Teórica. Revista Venezolana de Gerencia, 10(32), 675-691.

Eastlick, M. A. (1996). Consumer Intention to Adopt Interactive Teleshopping. Marketing Science Institute Working Paper (pp. 96-113). MA, Cambridge: Marketing Science Institute.

Eiglier, P., \& Langeard, E. (1993). Servucción. El Marketing de Servicios. España, Madrid: McGraw-Hill.

Fischer, E., Gainer, B., \& Bristor, J. (1997). The Sex of the Service Provider: Does it Influence Perceptions of Service Quality?. Journal of Retailing, 73(3), 361-382. http://dx.doi.org/10.1016/S0022-4359(97)90023-3 
Fisk, R. P., Brown, S. W., \& Bitner, M. J. (1993). Tracking the Evolution of the Services Marketing Literature. Journal of Retailing, 69(1), 61-103. http://dx.doi.org/10.1016/S0022-4359(05)80004-1

Flipo, J. P. (1993). Gestión de Empresas de Servicios. Barcelona: Gestión 2000.

Gil, I., Sánchez, M., Berenguer, G., \& González, M. (2005). Encuentro de Servicio, Valor Percibido y Satisfacción del Cliente en la Relación entre Empresas. Cuadernos de Estudios Empresariales, 15, 47-72.

Globerson, Shlomo, \& Maggard, Michael J. (1991). A Conceptual Model of Self-service., International journal of Operations and Production Management, 11(4), 33-43. http://dx.doi.org/10.1108/01443579110002977

González, J. L. (1990a). El Iceberg de la Política de Customer Satisfaction. MK Marketing y Ventas para directivos, 38, Junio.

González, J. L. (1990b). Customer Satisfaction: de la siembra a la cosecha. MK Marketing y Ventas para directivos, 39 , Julio.

Goodwin, C. (1996). Communality as a Dimension of Service Relationships. Journal of Consumer Psychology, 5(4), 387-415. http://dx.doi.org/10.1207/s15327663jcp0504_04

Goodwin, C., \& Gremler, D. D. (1996). Friendship Over the Counter: How Social Aspects of Service Encounters Influence Consumer Service Loyalty. En Swartz, T.A., Bowen, David E., \& Brown, Stephen W. (Eds.). Advances in Services Marketing and Management (vol. 5, pp. 247-82). CT, Greenwich: JAl Press.

Greco, A. J., \& Fields, D. M. (1991). Profiling Early Triers of Service Innovations: A Look at Interaction Home Video Ordering Services. Journal of Services Marketing, 5(3), 19-26.

http://dx.doi.org/10.1108/08876049110035585

Gremler, D. D., Gwinner, K. P., \& Brown, S. W. (2001). Generating Positive Word-of- Mouth Communication through Customer-Employee Relationships. International journal of Service Industry Management, 12(1), 44-59. http://dx.doi.org/10.1108/09564230110382763

Grove, S. J., \& Fisk, R. P. (1997). The Impact of Other Customers on Service Experiences: A Critical Incident Examination of 'Getting Along'. Journal of Retailing, 73(1), 217-224.

Hartline, M. D., \& Ferrell, O.C. (1996). The Management of Customer-Contact Service Employees: An Empirical Investigation. Journal of Marketing, 60, October, 52-70. http://dx.doi.org/10.2307/1251901

Harvey, J., Lefebvre, L. A., \& Lefebvre, E. (1997). Flexibility and Technology in Services: a Conceptual Model. International Journal Operations \& Production Management, 17(1), 29-45.

http://dx.doi.org/10.1108/01443579710157970

Herzberg, F. (1977). Una vez más: Cómo Motivar a los Trabajadores. Harvard-Deusto.

Heskett, J. L. (1987). Lecciones en el Sector de los Servicios. Harvard-Deusto Business Review, 40 trimestre. Heskett, J. L. (1988). La Gestión en las Empresas de Servicios. Barcelona: Plaza \& Janés Editores.

Hof, R. D. (1999, octubre 4). A New Era of Bright Hopes and Terrible Fears. BusinessWeek, pp. 84-98.

Hsieh, A.T., Yen, C.H., \& Chin, K.C. (2004). Participative Customer as Partial Employees and Service Provider Workload. International journal of Service Industry Management, 15(2), 187-199.

http://dx.doi.org/10.1108/09564230410532501

Huete, L. M., Roth, A. V., \& Vollmann, T. (1988). Delivery System Industrialization Strategies of American Retail Banks for 1990. Research Division-IESE, Barcelona, Noviembre.

Huselid, M. (1995). The Impact of Human Resource Management Practices on Turnover, Productivity, and Corporate Financial Performance. Academy If Management journal, 38, 635-672.

Johnston, R. (1989). The Customer as Employee. International Journal of Operations and Production Management, 9(5), 15-23. http://dx.doi.org/10.1108/EUM0000000001240

Juran, J. M. (1990). Juran y el Liderazgo para la Calidad. Un manual para directivos. Ediciones Díaz de Santos.

Kalleberg, A. L., \& Moody, J. W. (1994). Human Resource Management and Organizational performance. American Behavioral Scientist, 37, 948-962. http://dx.doi.org/10.1177/0002764294037007007

Langeard, E., Bateson, J. E. G., Lovelock, C. H., \& Eiglier, P. (1981). Marketing of Services: Newinsights from Consumers and Managers. MA, Cambridge: Marketing Science Institute.

Larrea Angulo, P. (1991). Calidad de Servicio. Del marketing a la Estrategia. Madrid: Díaz de Santos. Lawrence, P., \& Karr, J. (1996). Technology Spending and Alliances: New Highs in Financial Services Firms. Journal of Retail Banking Services, 17(3), 45-52. 
Lewis, B. R., \& Entwistle, T. W. (1990). Managing the Service Encounter: A Focus on the Employee. International Journal of Service Industry Management, 1(3), 41-52.

http://dx.doi.org/10.1108/09564239010001136

Liljander, V., \& Strandvik, T. (1995). The Nature of Customer Relationships in Services. En Swartz, T.A., Bowen, D.E., \& Brown, S.W. (Eds.). Advances in Services Marketing and Management (vol. 4).CT, Greenwich: JAl Press.

Lovelock, C. H., \& Young, R. F. (1977). Marketing's Potential for Improving Productivity in Service Industries. Marketing Science Institute.

Lovelock, C. H., \& Young, R. F. (1979). Look to Customers to Increase Productivity. Harvard Business Review, 57(3), Mayo-junio, 168-178.

Maister, D. H. (1982a). Job Assignments Set the Pace in Professional Service Firms. Journal of Management Consulting, Enero.

Maister, D. H. (1982b). Balancing the Professional Service Firm. Sloan Management Review, Otoño.

Maister, D. H. (1985). The One-Firm Firm: What Makes it Successful. Sloan Management Review, Otoño.

Martin, C. L., \& Pranter, C. A. (1989). Compatibility Management: Customer-to-Customer Relationships in Service Environments. Journal of Services Marketing, 3, Summer, 6-15.

Martin, C.R., Horne, D.A., \& Chan, W.S. (2001). A Perspective on Client Productivity in Business-to-Business Consulting Services. International Journal of Service Industry Management, 12(2), 137-157.

Meuter, M. L., \& Bitner, M. J. (1998). Self-Service Technologies: Extending Service Frameworks and Identifying Issues for Research. En Dhruv Grewal, \& Connie Pechmann (Eds.). AMA Winter Educators' Conference Proceedings: Marketing Theory and Applications (vol. 9, pp. 12-19). American Marketing Association.

Meuter, M. L., Bitner, M.J., Ostrom, A.L., \& Brown, S.W. (2005). Choosing Among Alternative Service Delivery Modes: An Investigation of Costumer Trial of Self-Service Technologies. journal of Marketing, . 69, April, .6183.

Meuter, M. L., Ostrom, A. L., Bitner, M. J., \& Roundtree, R. I. (2003). The Influence of Technology Anxiety on Consumer Use and Experiences with Self-Service Technologies. Journal of Business Research, 56, 899-906. http://dx.doi.org/10.1016/S0148-2963(01)00276-4

Meuter, M. L., Ostrom, A. L., Roundtree, R. I., \& Bitner, M. J. (2000). Self-Service Technologies:

Understanding Customer Satisfaction with Technology-Based Service Encounters. Journal of Marketing, 64, July, 50-64. http://dx.doi.org/10.1509/jmkg.64.3.50.18024

Mills, P. K. (1986). Managing Service Industries, Ballinger, Cambridge, USA.

Mohr, L. A., \& Bitner, M. J. (1995). Process Factors in Service Delivery: What Employee Effort Means to Customers. en Swartz, T.A., Bowen, D.E., \& Brown, S.W. (Eds.). Advances in Services Marketing and Management (vol. 4, pp. 91-117). CT, Greenwich: JAl Press.

Normann, R. (1990). La Gestión en las Empresas de Servicios. Estrategia y liderazgo. Bilbao: Deusto.

Parasuraman, A. (1996). Understanding and Leveraging the Role of Customer Service in External, Interactive and Internal Marketing, paper presented at Frontiers in Services Conference, TN, Nahville, October.

Parasuraman, A. (1998). Customers' Confort Level with Technology-Based Services: An empirical Study, paper presented at AMA winter Educators' Conference, TX, Auxtin, February 21-24.

Patterson, A., \& Baron, S. (2010). Deviant employees and dreadful service encounters: customer tales of discord and distrust. Journal of Services Marketing, 24(6), $438-445$. http://dx.doi.org/10.1108/08876041011072555

Price, L. L., Arnould, E. J., \& Deibler, S. L. (1995). Consumers' Emotional Responses to Service Encounters. International Journal of Service Industry Management, 6(3), 34-63.

http://dx.doi.org/10.1108/09564239510091330

Proença, J.F., \& Rodrigues, M.A. (2011) A comparison of users and non-users of banking self-service technology in Portugal. Managing Service Quality, 21(2), $192-210$.

http://dx.doi.org/10.1108/09604521111113465

Quinn, J. B. (1996). The Productivity Paradox Is False: Information technology Improves Service Performance. En Swartz, T.A., Bowen, D.E., \& Brown, S.W. (Eds.). Advances in Services Marketing and Management (vol. 5, pp. 71-84). CT, Greenwich: JAl Press. 
Rafaeli, A. (1993). Dress and Behavoir of Customer Contact Employees: A Framework for Analysis. En Swartz, T.A., Bowen, D.E., \& Brown, S.W. (Eds.). Advances in Services Marketing and Management (vol. 4, pp. 175-211). CT, Greenwich: JAl Press.

Raub, A. C. (1981). Correlates of Computer Enxiety in College Students. Doctoral dissertation, Universsity of Pennsylvania, Chemistry Department.

Rayport, J. F., \& Sviokla, J. J. (1995). Exploiting the Virtual Value Chain. Harvard Business Review, 73, November-December, 14-24.

Sasser, W. E. et al. (1982). Management of Service Operations. Boston: Allyn and Bacon.

Sasser, W. E., Olsen, R. P., \& Wyckoff, D. D. (1978). Management of Service Operation. Texts, cases and readings. Boston: Allyn and Bacon Inc.

Schneider, B., \& Bowen, D. E. (1985). Employee and Customer Perceptions of Service in Bands: Replication and Extension. Journal of Aplied Psychology, 70, 423-433. http://dx.doi.org/10.1037/0021-9010.70.3.423

Schneider, B., \& Bowen, D. E. (1995). Winning The Service Game. Boston: Harvard Business School Press.

Solomon, M. R., Surprenant, C., Czpiel, J. A., \& Gutman, E. G. (1985). A Role Theory Perspective on Dynamic Interactions: The Service Encounter. Journal of Marketing, 49, 99-111. http://dx.doi.org/10.2307/1251180

Suprenant, C. F., \& Solomon, M. R. (1987). Predictability and Personalization in the Service Encounter. journal of Marketing, 51, April, 73-80.

Tansik, D. A. (1990). Managing Human Resource Issues for High-Contact Service Personnel. En Bowen, D.E., Chase, R.B., Cummings, T.G., \& Associates. Service Management Effectiveness. Balancing Strategy, Organization and Human Resources, Operations, and Marketing (pp. 152-176). USA: Jossey-Bass Publishers.

Tat Keh, H., \& Wei Teo, C. (2001). Retail Customers as Partial Employees in Service Provision: A Conceptual Framework. International journal of Retail and Distribution Management, 29(8), 370-378. http://dx.doi.org/10.1108/09590550110396944

Toffler, A. (1980). The Thhird Wave, Collins.

Voss, C. A., Armistead, C. G., Johnston, R., \& Morris, B. (1985). Operations Management in Service Industries and the Public Sector. Chichester: Wiley.

Wägar, K., \& Lindqvist, L.J. (2010). The Role of the Customer Contact Person's Age in Service Encounters. Journal of Services Marketing, 24(7), 509 - 517. http://dx.doi.org/10.1108/08876041011081069

Walker, R. H., Craig-Lees, M., Hecker, R., \& Francis, H. (2002). Technology-Enabled Service Delivery: An Investigation of Reasons Affecting Customer Adoption and Rejection. International Journal of Service Industry Management, 13(1), 91-110. http://dx.doi.org/10.1108/09564230210421173

Zeithaml, V., \& Gilly, M. C. (1987). Characteristics Affecting the Acceptance of Retailing Technologies: A Comparison of Elderly and Nonelderly Consumers. Journal of Retailing, 63(1), 49-68. 